\title{
The future of helioseismology
}

\author{
Alexander G. Kosovichev \\ Hansen Experimental Physics Laboratory, Stanford University, Stanford, CA 94305, USA \\ email: AKosovichev@solar.stanford.edu
}

\begin{abstract}
Helioseismology has provided us with the unique knowledge of the interior structure and dynamics of the Sun, and the variations with the solar cycle. However, the basic mechanisms of solar magnetic activity, formation of sunspots and active regions are still unknown. Determining the physical properties of the solar dynamo, detecting emerging active regions and observing the subsurface dynamics of sunspots are among the most important and challenging problems. The current status and perspectives of helioseismology are briefly discussed.
\end{abstract}

Keywords. Sun: helioseismology, activity, interior magnetic fields, oscillations, sunspots

\section{Outstanding problems of helioseismology}

One of the most important unsolved problems of solar physics and astrophysics is understanding of the physical mechanism of the dynamo operating inside the Sun and producing 11-year activity cycles. Despite a significant progress in theoretical modeling of dynamo processes and successful laboratory experiments the basic understanding of the solar dynamo is still missing. The current theories assume that the poloidal component of magnetic field, which represents the global dipole field, is generated by helical turbulence at the bottom of the convection zone tachocline, and that the toroidal component, which is a source of bipolar sunspot regions, is produced by stretching the poloidal field by the differential rotation (e.g. Jouve, et al. 2008). However, so far helioseismology observations were not able to confirm these models. If the magnetic field is generated by turbulence it is expected that when the field becomes sufficiently strong it suppresses the turbulence and affects the turbulent stresses that maintain the differential rotation. However, the helioseismic observations during a whole solar cycle were not able to detect significant variations of the rotation rate in the tachocline. They found a weak evidence of variations with a period of $\sim 1.3$ years but no changes on the 11-year scale. In addition, to explain the emergence of magnetic fields at mid- and low latitudes the strength of the toroidal magnetic field in the tachocline must be $60-100 \mathrm{kG}$. This exceeds the energy equipartition value, and requires a dynamic compression, e.g. by back reaction of updraft motions associated with emerging magnetic flux (Parker, 2009). Such motions have not been detected by helioseismology. Alternatively, the magnetic fields can be generated in the bulk of the convection zone and shaped by the subsurface shear layer (Brandenburg, 2009). The equator-ward migration of dynamo waves in this shear layer could explain the sunspot butterfly diagram. The migrating zone of sunspot formations is associated with zonal flows ("torsional oscillations"). The magnetic flux that forms sunspots tend to emerge in the shear layer between slower and faster flows. Helioseismology has established that these flows are quite deep and occupy at least the upper $30 \%$ of the convection zone. They are particularly strong at high latitudes, where they migrate towards the poles, and perhaps are related to the polar field reversals. However, the physical mechanism of these flows and their relation to the dynamo mechanism are not yet established (Howe et al. 2009). Synoptic observations showed that the polar field reversals correlate with the polarward transport of magnetic flux. This transport is assumed to be caused by meridional flow. Local helioseismology has detected this flow below the surface, and found that its speed significantly changes with time (e.g. Svanda, 2007). The flux-transport dynamo theories assume that after reaching the polar regions the magnetic flux is transported downward to the tachocline, and then by a reverse meridional flow towards the low-latitude regions. However, 
the reverse flow has not been detected. To determine the deep structure of the Sun's meridional circulation is one of the greatest challenges. Successful imaging of the tachocline structure by time-distance helioseismology provides optimism that this problem will be solved (Zhao et al. 2009). Implementation of modern data assimilation methods (Kitiashvili et al. 2008) gives a promising approach for forecasting the state of the solar dynamo and future solar activity.

Another outstanding problem of helioseismology is the detection of magnetic flux before it become visible on the surface. Local helioseismology has been successful in detecting large active regions on the far-side of the Sun, and this technique is becoming more robust (Hartlep et al. 2008). However, observations of emerging magnetic field below the surface are very difficult, because helioseismic signals for deep perturbations are weak, and in the upper convection zone the magnetic flux is emerging very fast, not giving enough time to accumulate a sufficient signalto-noise ratio in the measurements. Therefore, the current efforts are focused on finding changes in the convective flow patterns associated with the emerging flux. The initial measurements have provided an evidence of upflows prior the flux emergence (Komm, et al. 2009). However, more systematic studies are necessary. Also, initial attempts are made to find a relationship between the evolution of subsurface sound-speed perturbations and flows and the development of the magnetic structure of active regions on surface (Kosovichev 2009).

\section{Future helioseismology projects}

The Helioseismic and Magnetic Imager (HMI) of board of the Solar Dynamics Observatory, scheduled for launch in 2010, will focus on investigations of the physical mechanisms and links among the solar dynamo, development of active regions and the internal dynamics leading to flares and coronal mass ejections (Kosovichev et al. 2007). The HMI will provide an unprecedented amount of high-resolution helioseismology data. Developing efficient and robust procedures for acoustic imaging of the deep interior is a new challenge of helioseismology. Future helioseismology projects include also currently planned out-of-ecliptic space missions, Solar Orbiter and Solar-C, which have a great potential for studying the solar dynamics in highlatitude and polar regions and for stereo-helioseismology of the tachocline and deeper interior. In addition, it is crucial to continue operating the ground-based helioseismology networks, GONG and BiSON, for monitoring the long-term behavior of our nearest star.

\section{References}

Brandenburg, A. 2009, in: Cosmic Magnetic Fields, Proc. IAU Symp., vol. 259, p. 159

Hartlep, T., et al. 2008, ApJ, 689, 1373

Howe, R., et al. 2009, ApJ, 701, L87

Jouve, L., et al. 2008, AछA, 483, 949

Kitiashvili, I. \& Kosovichev, A. G. 2008, ApJ, 688, L49

Komm, R., et al. 2009, Solar Phys., 258, 13

Kosovichev, A. G. \& HMI Science Team, 2007, Astr. Nach., 328, 339

Kosovichev, A. G. 2009, Space Science Rev., 144, 175

Parker, E. N. 2009, Space Science Rev., 144, 15

Švanda, M., et al. 2007, ApJ, 670, L69

Zhao, J., et al. 2009, ApJ, 702, 1150 